\title{
Recognition by character degree graph and order of simple groups of order less than 6000
}

\author{
Behrooz Khosravi, Bahman Khosravi, Behnam \\ Khosravi, and Zahra Momen
}




\title{
RECOGNITION BY CHARACTER DEGREE GRAPH AND ORDER OF SIMPLE GROUPS OF ORDER LESS THAN 6000
}

\author{
BEHROOZ KHOSRAVI, BEHNAM KHOSRAVI, BAHMAN KHOSRAVI, \\ AND ZAHRA MOMEN
}

Received 24 September, 2013

\begin{abstract}
Let $G$ be a finite group. The character degree graph of $G$, which is denoted by $\Gamma(G)$, is the graph whose vertices are the prime divisors of the character degrees of the group $G$ and two vertices $p_{1}$ and $p_{2}$ are joined by an edge if $p_{1} p_{2}$ divides some character degree of $G$. In this paper we prove that if $G$ is a simple group of order less that 6000 , then $G$ is uniquely determined by its character degree graph and its order. Also by an example we show that this result is not true for all simple groups.
\end{abstract}

2010 Mathematics Subject Classification: 20C15; 20D05; 20D60

Keywords: character degree graph, character degrees, order, simple group

\section{Introduction AND PRELIMINARy RESUlts}

Let $G$ be a finite group, $\operatorname{Irr}(G)$ be the set of irreducible characters of $G$, and denote by $\operatorname{cd}(G)$, the set of irreducible character degrees of $G$.

There are several graphs attached to the set of character degrees of a finite group that have been studied (see $[3,5])$. Results on these graphs are often useful to prove results that provide structural information of the group from some property of the set of character degrees. The graph that has been most commonly studied is the graph $\Gamma(G)$ whose vertices are the prime divisors of the character degrees of the group $G$ and two vertices are joined by an edge if the product of the primes divides some character degree of $G$. This graph is called character degree graph and was introduced in [5]. Later this graph has been widely studied (see [4,6]).

A finite group $G$ is called a $K_{3}$-group if $|G|$ has exactly three distinct prime divisors. Recently Chen et. al. in [7] proved that these groups are uniquely determined by their orders and one or both of their largest and second largest irreducible character degrees. It is proved that $A_{5}, L_{2}(7), L_{2}(17), L_{3}(3)$ and $U_{4}(2)$ can be uniquely determined by their orders and the largest degrees of their irreducible characters. Also it is proved that $A_{6}$ is characterized by its order and the second largest degree of

The first author was in part supported by grants from IPM (No 91050116 and No 92050120). 
its irreducible characters. Finally it is proved that $L_{2}(8)$ and $U_{3}(3)$ are characterizable by their orders and the largest and the second largest degrees of their irreducible characters.

The goal of this paper is to introduce a new characterization for the simple groups $S$ such that $|S|<6000$. In fact we prove the following result:

Let $S$ be a simple group such that $|S|<6000$. If $G$ is a finite group such that $|G|=|S|$ and $\Gamma(G)=\Gamma(S)$, then $G \cong S$.

We note that this result is not true for all simple groups. In fact we give a solvable group of order 6048 such that its order and its character degree graph are the same as $U_{3}(3)$ and so the simple group of order 6048 is not characterized by its degree graph and its order.

If $N \unlhd G$ and $\theta \in \operatorname{Irr}(N)$, then the inertia group of $\theta$ in $G$ is $I_{G}(\theta)=\{g \in G \mid$ $\left.\theta^{g}=\theta\right\}$. If the character $\chi=\sum_{i=1}^{k} e_{i} \chi_{i}$, where for each $1 \leq i \leq k, \chi_{i} \in \operatorname{Irr}(G)$ and $e_{i}$ is a natural number, then each $\chi_{i}$ is called an irreducible constituent of $\chi$.

Lemma 1 ((Ito's Theorem) [2, Theorem 6.15]). Let $A \unlhd G$ be abelian. Then $\chi(1)$ divides $|G: A|$, for all $\chi \in \operatorname{Irr}(G)$.

Lemma 2 ([2, Theorems 6.2, 6.8, 11.29]). Let $N \unlhd G$ and let $\chi \in \operatorname{Irr}(\mathrm{G})$. Let $\theta$ be an irreducible constituent of $\chi_{N}$ and suppose $\theta_{1}=\theta, \ldots, \theta_{t}$ are the distinct conjugates of $\theta$ in $G$. Then $\chi_{N}=e \sum_{i=1}^{t} \theta_{i}$, where $e=\left[\chi_{N}, \theta\right]$ and $t=\left|G: I_{G}(\theta)\right|$. Also $\theta(1) \mid \chi(1)$ and $\chi(1) / \theta(1)|| G: N \mid$.

Lemma 3 ([7, Lemma]). Let $G$ be a nonsolvable group. Then $G$ has a normal series $1 \unlhd H \unlhd K \unlhd G$ such that $K / H$ is a direct product of isomorphic nonabelian simple groups and $|G / K||| \operatorname{Out}(K / H) \mid$.

Lemma 4 ((Palfy's Theorem) [4, Theorem 4.1]). Let $\rho(G)$ be the set of all prime divisors of the character degrees of $G$. Let $G$ be a solvable group and $\pi \subseteq \rho(G)$. If $|\pi| \geq 3$, then there exist primes $p, q \in \pi$ and a degree $a \in \operatorname{cd}(G)$ so that $p q$ divides $a$. In other words, any three primes in $\rho(G)$ must have an edge in $\Gamma(G)$ that is incident to two of those primes.

If $n$ is an integer and $r$ is a prime number, then we write $r^{\alpha} \| n$, when $r^{\alpha} \mid n$ but $r^{\alpha+1} \nmid n$. Also if $r$ is a prime number we denote by $\operatorname{Syl}_{r}(G)$, the set of Sylow $r$ subgroups of $G$ and we denote by $n_{r}(G)$, the number of elements of $\operatorname{Syl}_{r}(G)$. If $H$ is a characteristic subgroup of $G$, we write $H \mathrm{ch} G$. All other notations are standard and we refer to [1].

\section{MAIN RESUltS}

Theorem 1. If $G$ is a finite group of order 60 such that $\Gamma(G)=\Gamma\left(A_{5}\right)$, then $G \cong A_{5}$.

Proof. We know that $\operatorname{cd}\left(A_{5}\right)=\{1,3,4,5\}$. Therefore $\Gamma(G)$ is a graph with vertex set $\{2,3,5\}$ and there is no edge in $\Gamma(G)$. So there exists $\chi \in \operatorname{Irr}(G)$, such that 
$5 \mid \chi(1)$. Also $\chi(1)^{2}<|G|=60$, implies that $\chi(1)=5$. On the other hand, the order and the character degree graph of $G$ show that 5 is the largest character degree of $G$. Therefore $G \cong A_{5}$, by [7].

Theorem 2. Let $G$ be a finite group of order 168 and $\Gamma(G)=\Gamma\left(L_{2}(7)\right)$. Then $G \cong L_{2}(7)$.

Proof. By [1], We know that $\operatorname{cd}\left(L_{2}(7)\right)=\{1,3,6,7,8\}$. Therefore $\Gamma(G)$ is a graph with vertex set $\{2,3,7\}$ and there exists an edge between 2 and 3 . Similarly to the proof of Theorem 1 there exists $\chi \in \operatorname{Irr}(G)$ such that $\chi(1)=7$. Also there exists $\beta \in \operatorname{Irr}(G)$ such that $6 \mid \beta(1)$. Therefore $\beta(1)=2^{\alpha} 3$, for some $\alpha>0$. Then $|G|=168$ shows that $\beta(1)=6$ or $\beta(1)=12$. If $\beta(1)=12$, then $1+\beta(1)^{2}+\chi(1)^{2}>|G|$, which is a contradiction.

If $O_{7}(G) \neq 1$, then $O_{7}(G)$ is an abelian normal subgroup of order 7 and we get a contradiction by Ito's theorem, since $\chi(1)=7$. Similarly it follows that $O_{3}(G)=1$.

Now we prove that $G$ is a nonsolvable group. On the contrary let $G$ be a solvable group. Let $M$ be a normal minimal subgroup of $G$. By the above discussion $M$ is a 2-elementary abelian subgroup of $G$. Also $|M| \neq 8$, by Ito's theorem, since $\beta(1)=6$. Hence $|M|=2^{k}$, where $1 \leq k \leq 2$. Hence if $Q / M \in \operatorname{Syl}_{7}(G / M)$, then $Q / M \triangleleft G / M$. Also if $P \in \operatorname{Syl}_{7}(Q)$, then $P$ ch $Q$, which implies that $P \triangleleft G$, and so $O_{7}(G) \neq 1$, which is a contradiction.

Therefore $G$ is nonsolvable and so $G$ has a normal series $1 \unlhd H \unlhd K \unlhd G$ such that $K / H$ is a direct product of isomorphic nonabelian simple groups and $|G / K| \mid$ $|\operatorname{Out}(K / H)|$. By the classification of finite simple groups and [1], it follows that $K / H$ is isomorphic to $L_{2}(7)$ and so $H=1$ and $G=K$. Therefore $G \cong L_{2}(7)$.

Theorem 3. Let $G$ be a finite group such that $|G|=\left|A_{6}\right|=2^{3} 3^{2} 5$ and $\Gamma(G)=$ $\Gamma\left(A_{6}\right)$. Then $G \cong A_{6}$.

Proof. By [1], We know that $\operatorname{cd}\left(A_{6}\right)=\{1,5,8,9,10\}$. Therefore $\Gamma(G)$ is a graph with vertex set $\{2,3,5\}$ and there is an edge between 2 and 5 . Similarly to the proof of Theorem 2 we conclude that $G$ has an irreducible character $\chi$ such that $\chi(1)=10$. Also by Ito's theorem it follows that $O_{5}(G)=1$.

Let $G$ be a solvable group and $M$ be a normal minimal subgroup of $G$. Then $M$ is a $p$-elementary abelian subgroup, where $p=2$ or $p=3$. Now we consider two cases:

Case 1. Let $M$ be a 3-group. Then $|M|=3$ or $|M|=9$. If $|M|=9$, then since 3 is a vertex of $\Gamma(G)$, we get a contradiction by Ito's theorem. Therefore $|M|=3$. Let $H / M$ be a Hall subgroup of $G / M$ of order $2^{3} 5$. Then $|G / M: H / M|=3$ and so $(G / M) /(L / M) \hookrightarrow S_{3}$, where $L / M=\operatorname{Core}_{G / M}(H / M)$. Therefore 5||$L / M \mid$ and $|L / M| \mid 40$, which implies that $Q / M \triangleleft L / M$, where $Q / M \in \operatorname{Syl}_{5}(L / M)$. Hence $Q \triangleleft G$ and $|Q|=15$. Therefore $O_{5}(G) \neq 1$, which is a contradiction.

Case 2. Let $M$ be a 2-group. Since $\chi(1)=10$, we get that $|M|=2$ or $|M|=4$.

(2.1) Let $|M|=4$. Then $|G / M|=2 \times 3^{2} \times 5$. 
Let $H / M$ be a Hall subgroup of $G / M$ of order $3^{2} 5$, which is a normal subgroup of $G / M$. Also $Q / M \triangleleft H / M$, where $Q / M \in \operatorname{Syl}_{5}(H / M)$, and so $Q / M \triangleleft G / M$. Therefore $Q \triangleleft G$ and $|Q|=20$. If $P \in \operatorname{Syl}_{5}(Q)$, then $P \triangleleft G$, which implies that $O_{5}(G) \neq 1$ and this is a contradiction.

(2.2) Therefore $|M|=2$ and $|G / M|=2^{2} 3^{2} 5$. Let $H / M$ be a Hall subgroup of $G / M$ of order $3^{2} 5$. Then $|G / M: H / M|=4$, and so if $L / M=\operatorname{Core}_{G / M}(H / M)$, then $(G / M) /(L / M) \hookrightarrow S_{4}$, which implies that 5||$L / M \mid$. Also $|L / M||| H / M \mid=$ $3^{2} 5$. Hence if $Q / M \in \operatorname{Syl}_{5}(L / M)$, then similarly to the above, $Q / M \triangleleft G / M$ and so $Q \triangleleft G$. Then $|Q|=10$ and if $P \in \operatorname{Syl}_{5}(Q)$, then $P \triangleleft G$, which implies that $O_{5}(G) \neq 1$, and this is a contradiction.

Therefore $G$ is nonsolvable and so $G$ has a normal series $1 \unlhd H \unlhd K \unlhd G$ such that $K / H$ is a direct product of isomorphic nonabelian simple groups and $|G / K| \mid$ $|\operatorname{Out}(K / H)|$. By the classification of finite simple groups and [1], it follows that $K / H$ is isomorphic to $A_{5}$ or $A_{6}$.

(i) Let $K / H \cong A_{5}$. Then $|H| \cdot|G / K|=6$ and $|G / K| \mid 2$.

(a) Let $|G / K|=1$. Then $|H|=6$. Now we consider two cases:

(a.1) Let $H \cong S_{3}$.

Then $H \triangleleft G$ and so $C_{G}(H) \triangleleft N_{G}(H)=G$. Also $H \cap C_{G}(H)=1$. Therefore $G \cong H \times C_{G}(H)$. Also $C_{G}(H) \cong G / H \cong A_{5}$ and hence $G \cong S_{3} \times A_{5}$. Now $G$ has an irreducible character of degree 6 , which is a contradiction, since 2 and 3 are nonadjacent in $\Gamma(G)$.

(a.2) Let $H \cong \mathbb{Z}_{6}$.

Then there exists $\theta \in \operatorname{Irr}(H)$ such that $e=\left[\chi_{H}, \theta\right] \neq 0$, where $\chi(1)=10$. Hence $10=e t$, where $t=\left|G: I_{G}(\theta)\right|$. We know that $t|| \operatorname{Aut}(H) \mid=2$. If $t=1$, then $e=10$, and so $\left[\chi_{H}, \chi_{H}\right]=e^{2} t=100<|G: H|=60$, which is a contradiction. Therefore $t=2$ and $e=5$. Since $H \subseteq I_{G}(\theta)$, it follows that $G / H \cong A_{5}$ has a subgroup $I_{G}(\theta) / H$ such that $\left|G / H: I_{G}(\theta) / H\right|=2$, and this is a contradiction.

(b) Let $|G / K|=2$. Then $G / H \cong S_{5}$ and we get a contradiction, since $S_{5}$ has an irreducible character of degree 6 .

(ii) Therefore $K / H \cong A_{6}$ and so $H=1$ and $G \cong A_{6}$.

Theorem 4. Let $G$ be a finite group such that $|G|=\left|L_{3}(3)\right|=2^{4} 3^{3} 13$ and $\Gamma(G)=\Gamma\left(L_{3}(3)\right)$. Then $G \cong L_{3}(3)$.

Proof. By [1], We know that $\Gamma(G)$ is a complete graph on the vertex set $\{2,3,13\}$. Then there exists $\chi \in \operatorname{Irr}(G)$, such that $\chi(1)=39$. So by Ito's theorem we get that $O_{13}(G)=1$.

Let $G$ be a solvable group. Let $M$ be a normal minimal subgroup of $G$. Then $M$ is a $p$-elementary abelian subgroup where $p=2$ or $p=3$. Since there exists $\beta \in \operatorname{Irr}(G)$ such that $6 \mid \beta(1)$, it follows that $|M| \neq 3^{3}$ and $|M| \neq 2^{4}$.

(i) Let $M$ be a 3-group. Then $|M|=3^{k}$, where $1 \leq k \leq 2$. Then $|G / M|=$ $2^{4} 3^{3-k} 13$. Let $H / M$ be a Hall subgroup of $G / M$ of order $2^{4} 13$. Then $\mid G / M$ : $H / M \mid=3^{3-k}$ and so $G / H_{G} \hookrightarrow S_{9}$ which implies that 13||$H_{G} \mid$. Let $Q / M \in$ 
$\operatorname{Syl}_{13}\left(H_{G} / M\right)$. Then $Q / M \triangleleft G / M$ and so $Q \triangleleft G$. If $P \in \operatorname{Syl}_{13}(Q)$, then $P$ ch $Q \triangleleft$ $G$, which implies that $O_{13}(G) \neq 1$, a contradiction.

(ii) Let $M$ be a 2-group and $|M|=2^{k}$, where $1 \leq k \leq 3$. Let $H / M$ be a Hall subgroup of $G / M$ of order $3^{3} 13$. Then $|G / M: H / M|=|G: H|=2^{r} \leq 8$, and so $G / H_{G} \hookrightarrow S_{8}$. Therefore 13||$H_{G} \mid$. Let $Q / M \in \operatorname{Syl}_{13}\left(H_{G} / M\right)$. Also $\left|H_{G} / M\right| \mid$ $|H / M|=3^{3} 13$. If $\left|H_{G} / M\right| \neq 3^{3} 13$, then $Q / M$ ch $H_{G} / M \triangleleft G / M$ and so $Q \triangleleft G$. Let $P \in \operatorname{Syl}_{13}(Q)$, where $|Q|=2^{k} 13,1 \leq k \leq 3$. Then $P \triangleleft G$, which implies that $O_{13}(G) \neq 1$ and this is a contradiction. Therefore $H_{G} / M=H / M$, i.e. $H / M \triangleleft$ $G / M$ and $|H|=2^{k} 3^{3} 13$. Let $\theta \in \operatorname{Irr}(H)$ such that $e=\left[\chi_{H}, \theta\right] \neq 0$. then $39=$ et $\theta(1)$, where $t=\left|G: I_{G}(\theta)\right|$. We know that $e$ and $t$ are divisors of $|G: H|=$ $2^{4-k}$. Therefore $e=t=1$ and so $\theta(1)=39$ and $\chi_{H}=\theta$. Since $\theta(1)^{2}=1521<$ $|H|$, it follows that $k=3$ and $|M|=8,|H|=2^{3} 3^{3} 13$. Hence $M \triangleleft G$ is a 2elementary abelian subgroup of $G$ of order 8 and $M \subseteq H$. Let $\eta \in \operatorname{Irr}(M)$ such that $e^{\prime}=\left[\theta_{M}, \eta\right] \neq 0$. Therefore $39=e^{\prime} t^{\prime}$, where $t^{\prime}=\left|H: I_{H}(\eta)\right|$. Also $M$ has 8 linear characters and so $t^{\prime} \leq 8$. Therefore one of the following occurs: $\left(e^{\prime}, t^{\prime}\right)=(13,3)$ or $(39,1)$. But in each case we have

$$
507=13^{2} 3 \leq\left[\theta_{M}, \theta_{M}\right]=e^{\prime 2} t^{\prime} \leq|H: M|=3^{3} 13,
$$

which is a contradiction. Therefore $G$ has a normal series $1 \unlhd H \unlhd K \unlhd G$ such that $K / H$ is a direct product of isomorphic nonabelian simple groups and $|G / K| \mid$ $|\operatorname{Out}(K / H)|$. By the classification of finite simple groups and [1], it follows that $K / H$ is isomorphic to $L_{3}(3)$. Hence $G \cong L_{3}(3)$.

Theorem 5. Let $q=8$ or 16 . Let $G$ be a finite group such that $|G|=\left|L_{2}(q)\right|$ and $\Gamma(G)=\Gamma\left(L_{2}(q)\right)$. Then $G \cong L_{2}(q)$.

Proof. Case 1. Let $q=8$.

We know that $\operatorname{cd}\left(L_{2}(8)\right)=\{1,7,8,9\}$. Therefore $\Gamma(G)$ is a graph with vertex set $\{2,3,7\}$ and there is no edge in $\Gamma(G)$. So by Lemma 4 we get that $G$ is a nonsolvable group. Therefore $G$ has a normal series $1 \unlhd H \unlhd K \unlhd G$ such that $K / H$ is a direct product of isomorphic nonabelian simple groups and $|G / K||| \operatorname{Out}(K / H) \mid$. By the classification of finite simple groups and [1], it follows that $K / H$ is isomorphic to $L_{2}(7)$ or $L_{2}(8)$. Since $6 \in \operatorname{cd}\left(L_{2}(7)\right)$, we get that $K / H \cong L_{2}(8)$ and so $G \cong L_{2}(8)$.

Case 2. Let $q=16$.

Similarly to Case 1, by Lemma 4 we get that $G$ is nonsolvable and we get that $G$ has a normal series $1 \unlhd H \unlhd K \unlhd G$ such that $K / H \cong A_{5}$ or $L_{2}$ (16). If $K / H \cong A_{5}$, then $|H| \cdot|G / K|=2^{2} 17$ and $|G / K| \mid 2$. Let $P \in \operatorname{Syl}_{17}(H)$. Then $P$ ch $H \triangleleft G$, which implies that $O_{17}(G) \neq 1$. Now we get a contradiction by Ito's theorem since 17 is a vertex of $\Gamma(G)$. Therefore $K / H \cong L_{2}(16)$ and so $G \cong L_{2}(16)$.

Theorem 6. Let $G$ be a finite group such that $|G|=\left|A_{7}\right|=2^{3} 3^{2} 5 \times 7$ and $\Gamma(G)=$ $\Gamma\left(A_{7}\right)$. Then $G \cong A_{7}$. 
Proof. The charcter degree graph of $A_{7}$ is a complete graph on the vertex set $\{2,3,5,7\}$. Also there exists $\chi \in \operatorname{Irr}(G)$, such that $21 \mid \chi(1)$.

Let $G$ be a solvable group. Then let $H$ be a Hall subgroup of $G$ of order $2^{3} 3^{2} 7$. Then $|G: H|=5$ and so $G / H_{G} \hookrightarrow S_{5}$. The order of a solvable subgroup of $S_{5}$ which is divisible by 5 is a divisor of 20 . Let $\theta \in \operatorname{Irr}\left(H_{G}\right)$ such that $\left[\chi_{H_{G}}, \theta\right] \neq 0$. Then $\chi(1)=e t \theta(1)$ and $e$ and $t$ are divisors of $\left|G: H_{G}\right|$, which implies that $e \mid 20$ and $t \mid 20$. Therefore $21 \mid \theta(1)$ and since $\theta(1)^{2}<\left|H_{G}\right|$, it follows that $\left|H_{G}\right|=504$ and so $H=H_{G} \triangleleft G$. On the other hand, there exists $\eta \in \operatorname{Irr}(G)$ such that $\eta(1)=35$. Let $\varphi \in \operatorname{Irr}(H)$ such that $\left[\eta_{H}, \varphi\right] \neq 0$. Then $35=\chi(1)=\operatorname{et} \varphi(1)$, where $\varphi(1)|| H \mid$ and et ||$G: H \mid=5$. Therefore $\theta(1)=7$ and $e t=5$. Since $\left[\eta_{H}, \eta_{H}\right]=e^{2} t \leq|G: H|$, it follows that $e=1$ and $t=5$. Hence $H$ has 5 irreducible characters of degree 7. Therefore $1+21^{2}+5 \times 7^{2} \leq|H|=504$, which is a contradiction. So $G$ is a nonsolvable group and by Lemma 3, $G$ has a normal series $1 \unlhd H \unlhd K \unlhd G$ such that $K / H$ is a direct product of isomorphic nonabelian simple groups and $|G / K| \mid$ $|\operatorname{Out}(K / H)|$. By the classification of finite simple groups and [1], it follows that $K / H$ is isomorphic to $A_{5}, A_{6}, A_{7}, L_{2}(7)$ or $L_{2}(8)$.

If $K / H \cong A_{5}$ or $A_{6}$, then 7||$H \mid$ and since $|H| \mid 42$, we get that $O_{7}(G) \neq 1$. Now by Ito's theorem we get a contradiction, since 7 is a vertex of $\Gamma(G)$.

If $K / H \cong L_{2}(7)$ or $L_{2}(8)$, then 5||$H \mid$ and $|H| \mid 15$, which implies that $O_{5}(G) \neq$ 1 and we get a contradiction by Ito's theorem.

Finally $K / H \cong A_{7}$ and so $G \cong A_{7}$.

Theorem 7. Let $p \in\{11,13,17,19\}$. If $G$ is a finite group such that $|G|=\left|L_{2}(p)\right|$ and $\Gamma(G)=\Gamma\left(L_{2}(p)\right)$. Then $G \cong L_{2}(p)$.

Proof. Since $p^{2} \nmid|G|$, and $p$ is a vertex in the character degree graph of $G$, by Ito's theorem it follows that $O_{p}(G)=1$.

Case 1. Let $p=11$. Then $|G|=2^{2} \times 3 \times 5 \times 11$.

If $G$ is solvable, let $H$ be a Hall subgroup of $G$ of order $2^{2} \times 5 \times 11$. Then $G / H_{G} \hookrightarrow S_{3}$. Hence 11||$H_{G} \mid$. Now if $P \in \operatorname{Syl}_{11}(G)$, then $P \triangleleft G$, which implies that $O_{11}(G) \neq 1$ and this is a contradiction. Therefore $G$ has a normal series $1 \unlhd H \unlhd K \unlhd G$ such that $K / H$ is isomorphic to $A_{5}$ or $L_{2}(11)$. If $K / H \cong A_{5}$, then $|H||G / K|=11$ and $|G / K| \mid 2$. Then $|H|=11$ and so $O_{11}(G) \neq 1$, which is a contradiction. Therefore $K / H \cong L_{2}(11)$ and so $G \cong L_{2}(11)$.

Case 2. Let $p=13$. Then if $G$ is a solvable group, let $H$ be a Hall subgroup of $G$ of index 7. Then 13||$H_{G} \mid$ and similarly to above $O_{13}(G) \neq 1$, which is a contradiction. Now similarly to above we get that $G \cong L_{2}(13)$.

Case 3. Let $p=17$. Then if $G$ is a solvable group, let $H$ be a Hall subgroup of $G$ of index 9. Then 17||$H_{G} \mid$ and similarly to the above $O_{17}(G) \neq 1$, which is a contradiction. Now similarly to the above we get that $G \cong L_{2}(17)$.

Case 4. If $p=19$, then similarly we get the result. 
Remark 1. The first simple group of order greater than 6000 is $U_{3}(3)$ of order 6048. In the sequel we show that this group is not characterizable by its character degree graph and its order. We know that the character degrees of $U_{3}(3)$ are $\{1,6,7,14,21,27,28,32\}$. So $\Gamma\left(U_{3}(3)\right)$ is a complete graph on the three vertices 2, 3 and 7. Now we construct a solvable group $G$ with $|G|=2^{5} 3^{3} \times 7$ and $\Gamma(G)=\Gamma\left(U_{3}(3)\right)$. Let $H$ be the Frobenius group of order $56=8 \times 7=2^{3} \times 7$, and observe that $\operatorname{cd}(H)=\{1,7\}$. Let $P$ be an extraspecial group of order $3^{3}$ and exponent 3. It is not difficult to see that $P$ has an automorphism $\sigma$ of order 2 that does not centralize the center of $P$. Let $K$ be the semi-direct product of $\langle\sigma\rangle$ acting on $P$. It is not difficult to see that $|K|=2 \times 3^{3}$ and $\operatorname{cd}(K)=\{1,2,6\}$. Let $Z$ be a cyclic group of order 2 , and take $G=H \times K \times Z$. Then $|G|=2^{5} 3^{3} \times 7$ and $\operatorname{cd}(G)=\{1,2,6,7,14,42\}$. Thus, $\Gamma(G)$ is a complete graph on three vertices.

\section{ACKNOWLEDGEMENT}

The authors would like to thank the referee for very valuable suggestions, specially in Remark 1, which improved the manuscript. The first author would like to thank the Institute for Research in Fundamental sciences (IPM) for the financial support.

\section{REFERENCES}

[1] J. H. Conway, R. T. Curtis, S. P. Norton, R. A. Parker, and R. A. Wilson, Atlas of finite groups. Oxford: Oxford University Press, 1985.

[2] I. M. Isaacs, Character theory of finite groups. New York: Academic Press, 1976.

[3] I. M. Isaacs, "Character degree graphs and normal subgroups," Trans. Amer. Math. Soc., vol. 356, no. 3, pp. 1155-1183, 2004.

[4] M. L. Lewis, "An overview of graphs associated with character degrees and conjugacy class sizes in finite groups," Rocky Mountain J. Math., vol. 38, no. 1, pp. 175-211, 2008.

[5] O. Manz, R. Staszewski, and W. Willems, "On the number of componenets of a graph related to character degrees," Proc. Amer. Math. Soc., vol. 103, pp. 31-37, 1988.

[6] D. L. White, "Degree graphs of simple groups," Rocky Mountain J. Math., vol. 39, no. 5, pp. 17131739, 2009.

[7] H. Xu, G. Y. Chen, and Y. Yan, "A new characterization of simple $k_{3}$-groups by their orders and large degrees of their irreducible characters," Comm. Algebra, to appear.

Authors' addresses

\section{Behrooz Khosravi}

School of Mathematics, Institute for Research in Fundamental sciences (IPM), P.O.Box: 193955746, Tehran, Iran

Current address: Dept. of Pure Math., Faculty of Math. and Computer Sci., Amirkabir University of Technology (Tehran Polytechnic), 424, Hafez Ave., Tehran 15914, Iran

E-mail address: khosravibbb@yahoo.com 


\section{BEHROOZ KHOSRAVI, BEHNAM KHOSRAVI, BAHMAN KHOSRAVI, AND ZAHRA MOMEN}

\section{Behnam Khosravi}

Iran

Department of Mathematics, Institute for Advanced Studies in Basic Sciences, Zanjan 45137-66731,

E-mail address: behnam_kho@yahoo.com

\section{Bahman khosravi}

Department of Mathematics, Faculty of Science, Qom University of Technology, Qom, Iran

E-mail address: bahman_kho@yahoo.com

\section{Zahra Momen}

Dept. of Pure Math., Faculty of Math. and Computer Sci., Amirkabir University of Technology (Tehran Polytechnic), 424, Hafez Ave., Tehran 15914, Iran

E-mail address: zahramomen@yahoo.com 